\title{
Crystal structure of tristrontium [tetranitridomolybdate(VI)], $\mathrm{Sr}_{3}\left[\mathrm{MoN}_{4}\right]$
}

\author{
P. Höhn* and R. Kniep \\ Max-Planck-Institut für Chemische Physik fester Stoffe, Pirnaer Landstr. 176, D-01257 Dresden, Germany
}

Received December 22, 1999, CSD-No. 409473

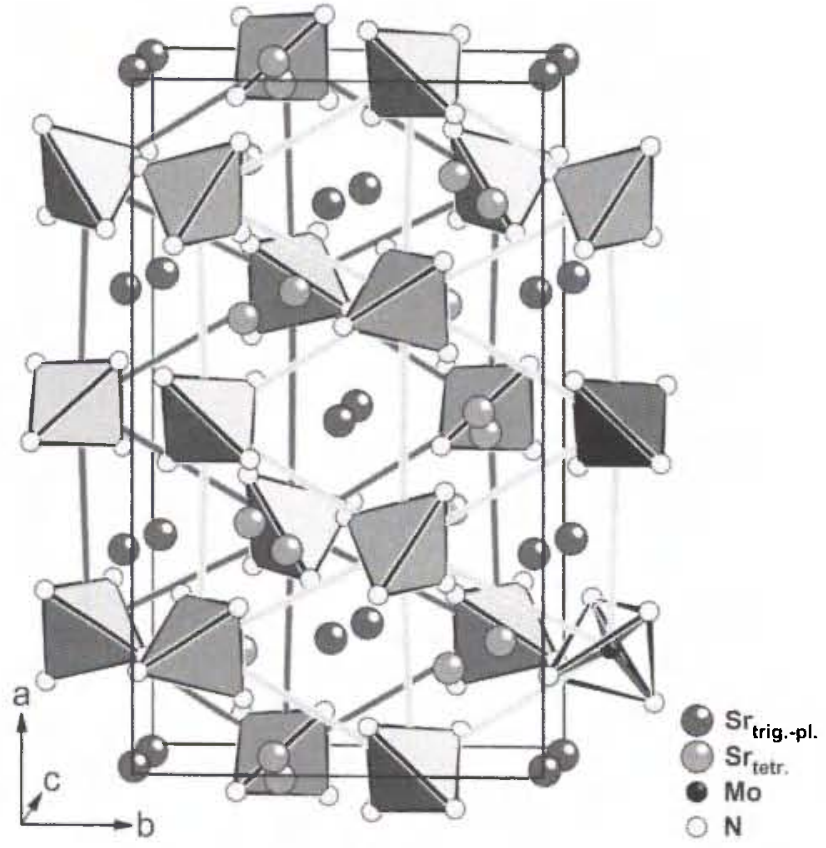

\section{Discussion}

The crystal structures of several ternary alkaline earth nitridomolybdates and -tungstates with $\mathrm{A}_{3}\left[\mathrm{MN}_{4}\right]$ composition are known up to now: the isotypes $\mathrm{LT}-\mathrm{Ba}_{3}\left[\mathrm{MoN}_{4}\right]$ [1] and LT-Ba $\left[\mathrm{WN}_{4}\right]$ [1], the isotypes HT-Ba3 $\left[\mathrm{MoN}_{4}\right][2,3]$ and HT-Ba3 $\left[\mathrm{WN}_{4}\right][2,4]$, and $\mathrm{Ca}_{2} \mathrm{Sr}\left[\mathrm{WN}_{4}\right]$ [5]. Predominant structural features of these compounds are isolated complex tetrahedral nitridomolybdate and nitridotungstate anions $\left[\mathrm{MN}_{4}\right]^{6-}$. The above mentioned crystal structures are related to the $\mathrm{Na}_{3} \mathrm{As}$-type structure with the complex anions in a hexagonal close packing arrangement. The tetrahedral holes are occupied by $2 / 3$ of the alkaline earth cations, the remaining alkaline earth cations are in a trigonal planar coordination by the complex anions. The crystal structures of the isotypes $\mathrm{Sr}_{3}\left[\mathrm{MoN}_{4}\right]$ and $\mathrm{Sr}_{3}\left[\mathrm{WN}_{4}\right]$ [2] are also members of this structure family; the most obvious differences between the different crystal structures refer to the orientations of the complex anions and the resulting differences in nitrogen coordination polyhedra around the alkaline earth cations. Mo is tetrahedrally coordinated by $\mathrm{N}(d(\mathrm{Mo}-\mathrm{N})=1.867 \AA)$, the coordination polyhedra of $\mathrm{Sr}$ are irregular $(\mathrm{CN}: 5-7 ; d(\mathrm{Sr}-\mathrm{N})=$ $2.757 \AA$ ). Both values are in good agreement with data from literature: $d(\mathrm{Mo}-\mathrm{N})=1.867 \AA\left(\mathrm{LT}-\mathrm{Ba}_{3}\left[\mathrm{MoN}_{4}\right][1], 1.863 \AA\right.$ $\left(\mathrm{HT}-\mathrm{Ba}_{3}\left[\mathrm{MoN}_{4}\right][3]\right) ; d(\mathrm{Sr}-\mathrm{N})=2.733 \AA\left(\mathrm{Ca}_{2} \mathrm{Sr}\left[\mathrm{WN}_{4}\right][5]\right)$.

\footnotetext{
Abstract

$\mathrm{MoN}_{4} \mathrm{Sr}_{3}$, monoclinic, $C 12 / c 1$ (No. 15), $a=17.793(1) \AA$, $b=10.295(1) \AA, c=9.891(1) \AA, \beta=97.75(2)^{\circ}, V=1795.3 \AA^{3}$, $Z=12, R_{\mathrm{gt}}(F)=0.045, w R_{\mathrm{ref}}\left(F^{2}\right)=0.112, T=293 \mathrm{~K}$.

\section{Source of material}

Yêllow single crystals were obtained by reaction of pellets of $\mathrm{Sr}_{2} \mathrm{~N}$ and $\mathrm{Mo}$ (molar ratio 4:1). The reaction was carried out under $\mathrm{N}_{2}$ at ambient pressure in Mo crucibles. The mixtures were heated to $1323 \mathrm{~K}(100 \mathrm{~K} / \mathrm{h})$. After a period of $36 \mathrm{~h}$ the products were cooled down $(20 \mathrm{~K} / \mathrm{h})$ to ambient temperature. Starting materials and products are sensitive to moisture and air.
}

Table 1. Data collection and handling.

$\begin{array}{ll}\text { Crystal: } & \begin{array}{l}\text { yellow, parallelepiped, } \\ \text { size } 0.4 \times 0.2 \times 0.1 \mathrm{~mm}\end{array} \\ & \text { Mo } K_{\alpha} \text { radiation }(0.71070 \AA) \\ \text { Wavelength: } & 285.27 \mathrm{~cm}^{-1} \\ \mu: & \text { Philips PW } 1100, \omega / 2 \theta \\ \text { Diffractometer, scan mode: } & 59.98^{\circ} \\ 2 \theta_{\text {max: }} & 4440,2169 \\ N(h k l)_{\text {measured, }}, N(h k l)_{\text {unique: }}: & I_{\text {obs }}>2 \sigma\left(I_{\text {obs }}\right), 1993 \\ \text { Criterion for } I_{\text {obs }}, N(h k l)_{\text {gr: }}: & 111 \\ N(\text { param })_{\text {refined: }} & \text { SHELXS-97 [6], SHELXL-97 [6], } \\ \text { Programs: } & \text { DIAMOND [7] }\end{array}$

Table 2. Atomic coordinates and displacement parameters (in $\hat{\AA}^{2}$ ).

\begin{tabular}{|c|c|c|c|c|c|c|c|c|c|c|}
\hline Atom & Site & $x$ & $y$ & $z$ & $U_{11}$ & $U_{22}$ & $U_{33}$ & $U_{12}$ & $U_{13}$ & $U_{23}$ \\
\hline $\operatorname{Sr}(1)$ & $4 e$ & $1 / 2$ & $0.4910(1)$ & $1 / 4$ & $0.0056(5)$ & $0.0102(5)$ & $0.0172(6)$ & 0 & $0.0023(5)$ & 0 \\
\hline $\operatorname{Sr}(2)$ & $8 f$ & $0.02302(5)$ & $0.33899(8)$ & $0.08772(9)$ & $0.0089(4)$ & $0.0108(4)$ & $0.0065(4)$ & $-0.0023(3)$ & $0.0017(3)$ & $0.0002(3)$ \\
\hline $\operatorname{Sr}(3)$ & $8 f$ & $0.34665(5)$ & $0.26625(7)$ & $0.40018(8)$ & $0.0063(4)$ & $0.0069(3)$ & $0.0057(4)$ & $0.0002(3)$ & $0.0009(3)$ & $-0.0004(3)$ \\
\hline $\operatorname{Sr}(4)$ & $8 f$ & $0.32278(5)$ & $0.37283(7)$ & $0.04567(8)$ & $0.0066(4)$ & $0.0071(3)$ & $0.0053(4)$ & $0.0003(3)$ & $0.0007(3)$ & $0.0000(3)$ \\
\hline $\operatorname{Sr}(5)$ & $8 f$ & $0.18546(5)$ & $0.46737(8)$ & $0.29985(9)$ & $0.0076(4)$ & $0.0073(4)$ & $0.0131(4)$ & $-0.0004(3)$ & $-0.0010(3)$ & $0.0010(3)$ \\
\hline $\operatorname{Mo}(1)$ & $4 e$ & $1 / 2$ & $0.16572(9)$ & $1 / 4$ & $0.0038(5)$ & $0.0056(4)$ & $0.0057(5)$ & 0 & $0.0002(4)$ & 0 \\
\hline $\operatorname{Mo}(2)$ & $8 f$ & $0.17573(4)$ & $0.14820(7)$ & $0.28766(7)$ & $0.0037(3)$ & $0.0048(3)$ & $0.0040(3)$ & $0.0001(2)$ & $0.0009(3)$ & $-0.0001(2)$ \\
\hline
\end{tabular}


Table 2. Continued.

\begin{tabular}{rllllllrrrrr}
\hline Atom & Site & $x$ & $y$ & $z$ & $U_{11}$ & $U_{22}$ & $U_{33}$ & $U_{12}$ & $U_{13}$ & $U_{23}$ \\
\hline $\mathrm{N}(1)$ & $8 f$ & $0.2407(4)$ & $0.0959(7)$ & $0.4432(8)$ & $0.005(3)$ & $0.012(3)$ & $0.007(3)$ & $-0.002(3)$ & $0.005(3)$ & $0.003(3)$ \\
$\mathrm{N}(2)$ & $8 f$ & $0.1399(5)$ & $0.0031(7)$ & $0.1907(8)$ & $0.017(4)$ & $0.008(3)$ & $0.007(4)$ & $-0.002(3)$ & $0.001(3)$ & $-0.005(3)$ \\
$\mathrm{N}(3)$ & $8 f$ & $0.2336(5)$ & $0.2478(7)$ & $0.1836(8)$ & $0.015(4)$ & $0.009(3)$ & $0.007(3)$ & $-0.005(3)$ & $0.007(3)$ & $0.001(3)$ \\
$\mathrm{N}(4)$ & $8 f$ & $0.0966(5)$ & $0.2519(8)$ & $0.3360(8)$ & $0.011(4)$ & $0.014(4)$ & $0.013(4)$ & $0.004(3)$ & $-0.004(3)$ & $-0.001(3)$ \\
$\mathrm{N}(5)$ & $8 f$ & $0.4348(5)$ & $0.0665(7)$ & $0.3458(8)$ & $0.017(4)$ & $0.008(3)$ & $0.014(4)$ & $-0.002(3)$ & $0.006(3)$ & $0.002(3)$ \\
$\mathrm{N}(6)$ & $8 f$ & $0.4491(5)$ & $0.2789(7)$ & $0.1239(8)$ & $0.018(4)$ & $0.011(4)$ & $0.013(4)$ & $0.006(3)$ & $0.002(3)$ & $-0.003(3)$ \\
& & & & & & & & & & & \\
\hline
\end{tabular}

Acknowledgments. We thank the Deutsche Forschungsgemeinschaft and the Fonds der Chemischen Industrie for financial support of this work.

\section{References}

1. Gudat, A.; Höhn, P.; Kniep, R.; Rabenau, A.: The crystal structures of the isotypic compounds $\mathrm{Ba}_{3}\left[\mathrm{MoN}_{4}\right]$ and $\mathrm{Ba}_{3}\left[\mathrm{WN}_{4}\right]$. Z. Naturforsch. 46b (1991) 566-572.

2. Höhn, P.; Gudat, A.; Kniep, R.; Maier, J.: Preparation and crystal chemistry of nitridomolybdates (VI) and nitridotungstates (VI). ECSSC IV, A 56, Dresden, Germany 1992.

3. Höhn, P.; Kniep, R.: Crystal structure of the high temperature phase of tribarium [tetranitridomolybdate(VI)], $\mathrm{HT}-\mathrm{Ba}_{3}\left[\mathrm{MoN}_{4}\right]$. Z. Kristallogr. NCS 215 (2000) 327-328.
4. Höhn, P.; Kniep, R.: Crystal structure of the high temperature phase of tribarium [tetranitridotungstate(VI)], HT-Ba3 $\left[\mathrm{WN}_{4}\right]$. Z. Kristallogr. NCS 215 (2000) 329-330.

5. Berger, U.; Schultz-Coulon, V.; Schnick, W.: $\mathrm{Ca}_{2} \mathrm{Sr}\left[\mathrm{WN}_{4}\right]$, the first mixed alkaline earth nitridotungstate. Z. Naturforsch. 50b (1995) 213-216.

6. Sheldrick, G. M.: SHELXL-97, a program for refining crystal structures. University of Göttingen, Germany 1997.

7. Brandenburg, K.: Diamond (Version 2.1a). Crystal Impact GbR, Germany 1996-1999. 